\title{
Modeling of fiber-optic fluorescence probes for strongly absorbing samples
}

\author{
Dorit Munzke, ${ }^{1}$ John Saunders, ${ }^{2}$ Hengameh Omrani, ${ }^{2}$ Oliver Reich, ${ }^{1}$ \\ and Hans-Peter Loock ${ }^{2, *}$ \\ 'innoFSPEC, Institute for Chemistry, Physical Chemistry, University of Potsdam, Am Mühlenberg 3, \\ D-14476 Potsdam-Golm, Germany \\ ${ }^{2}$ Department of Chemistry, Queen's University, 90 Bader Lane, Kingston, Ontario K7L 3N6, Canada \\ ${ }^{*}$ Corresponding author: hploock@chem.queensu.ca
}

Received 20 June 2012; revised 3 August 2012; accepted 5 August 2012;

posted 7 August 2012 (Doc. ID 170948); published 7 September 2012

\begin{abstract}
The dynamic range of fiber-optic fluorescent probes such as single fibers and fiber bundles is calculated for strongly absorbing samples, such as process liquids, foodstuffs, and lubricants. The model assumes an excitation beam profile based on a Lambertian light source and uses analytical forms of the collection efficiency, followed by an Abel transformation and numerical integration. It is found that the effect of primary absorption of the excitation light and secondary absorption of the fluorescence is profound. For fiber bundles and bifurcated fiber probes, the upper accessible concentration limit is roughly given by the absorption length of the primary and secondary absorption. Fluorescence detectors that are placed at right angles to the excitation beam axis or collinear to the beam axis are equally strongly affected by secondary absorption. A probe in which the same fiber is used for excitation and for collection of the fluorescence emerges as the fiber probe with the largest accessible concentration range. (C) 2012 Optical Society of America
\end{abstract}

OCIS codes: $\quad 060.2370,170.6280,300.6280,220.2740$.

\section{Introduction}

Fiber-optic probes are frequently used as a convenient means to excite and collect fluorescence in a variety of condensed media. Fluorescing sample media include foodstuffs [1] , human tissue [2-4] , and environmental contaminants such as polycyclic aromatic hydrocarbons [5], as well as lubricants, fuels, and other industrial liquids [6-8]. The use of silica fiber probes together with fluorescence excitation-emission matrix (EEM) spectra allows for the collection of twodimensional data arrays over large excitation and emission wavelength ranges. After calibration and multiway analysis, these spectra frequently contain enough information that identification and

$1559-128 \mathrm{X} / 12 / 266343-09 \$ 15.00 / 0$

(C) 2012 Optical Society of America quantification of the chemical composition of an unknown sample becomes possible.

In general, a fluorescing sample may strongly absorb light. The absorption of the excitation light and the reabsorption of the emitted fluorescence are therefore important considerations when designing fiber-optic probes, since they limit the volume of liquid that produces detectable fluorescence. In this paper, we compare four typical experimental configurations that are used to excite and collect fluorescence in absorbing media. We identify key design parameters in an attempt to arrive at fiber-optic probe configurations that permit the largest measurable range of concentrations and absorptivities.

For applications in process monitoring, assessment of lubricant and fuel quality, environmental assessment, and food quality assurance, the samples need to be investigated in situ and undiluted. Strong fluorescence then frequently is accompanied by 
strong scattering and absorption. An ideal probe can be used to collect fluorescence EEM spectra following excitation of strong absorption bands with respective strong reabsorption of the fluorescence signal, but it can also be used to excite weakly absorbing spectral features and detect faint fluorescence emission. In samples such as industrial lubricants, the absorption coefficients and the corresponding fluorescence emission intensity spans 4 to 5 orders of magnitude in the UV-Vis-NIR spectrum, and only a probe with a large dynamic range is capable of capturing the information contained in all spectral features [8]. Light scattering further complicates modeling of the sensor response and, while important, especially for food and tissue samples, will be neglected in the present study.

Recent work on optimization of fiber probes for medical samples has highlighted the need to collect the fluorescence (or Raman signal) before it is attenuated by the sample. Configurations include simple bifurcated fibers [9] and fiber bundles in which the fiber ends are angled toward each other [10], beveled to refract the fluorescence into the fiber [2,11-13], or coupled to lenses $[11,12]$. The gaps between the collection fibers in a fiber bundle are avoided, and the collection efficiency is further increased, when a coaxial design is used. Here the excitation fiber is surrounded by a high-index capillary that is capable of collecting and guiding the fluorescence to the detector [14]. For the same purpose, a double-clad fiber has also been used previously [15]. In an alternative design, the fiber(s) that excite the fluorescence and the fiber(s) that collect fluorescence are placed at some distance from the sample to ensure that the entire fluorescence volume falls into the field of view of the collection fibers [2]. The effect of the target distance on the collection efficiency was the subject of a theoretical Monte Carlo simulation [16], which was combined with an experimental study.

Modeling has been used extensively to quantify the effect of this dead volume. For example, Ma et al. [9] derived an analytical expression that permits the calculation of the fluorescence intensity as a function of the core and cladding radius of the adjacent excitation and the collection fiber in a bifurcated fiber probe. Other studies examined the effect of the distance between a planar fluorescence emitter and the end of a bifurcated fiber probe [15] or different designs of fiber bundles [16] using Monte Carlo simulations. Almost all previous modeling studies have assumed that the fluorescence medium has negligible absorption $[9,11]$ or that it is planar $[16,17]$. These studies were therefore not designed to quantify the effect of primary absorption of the excitation light and secondary absorption of the emitted fluorescence. The (re-) absorption of light may be neglected when the sample is very dilute or in the gas phase $[18,19]$ but cannot be ignored in many undiluted liquid samples. An experimental study by Ozanyan et al. [20] was supplemented with numerical modeling of the light emission from a single-fiber probe and a bifurcated probe to illustrate the effect of the dead volume on strongly attenuating samples. These authors explicitly included attenuation of the sample and also examined the effect of the fiber's numerical aperture. Despite the different models used for the light source, their main results are comparable to ours.

To the best of our knowledge, in all the previous simulations the intensity distribution of a multimode fiber was assumed to be a flat-topped distribution $[9,16,20,21]$ that expands according to the numerical aperture of the fiber and the refractive index of the sample. The assumption is likely idealized but frequently permits a closed analytical treatment. Still, for a lamp or LED as a light source, a flat-topped distribution or even a Gaussian or Lorentzian distribution may not be the best representation of the multimode-fiber emission cone.

The present study was performed as part of an attempt to design a fiber-optic detection system optimized for strongly absorbing machinery fluids such as lubricants and fuel. The need to address the effects of primary and secondary absorption in fluorescence EEM spectroscopy became apparent when we attempted to examine neat jet engine turbine oil, a sample that exhibits both strong absorption bands at $\lambda<300 \mathrm{~nm}$ and much weaker absorption features at $\lambda>500 \mathrm{~nm}$, with correspondingly strong and weak contributions of primary and secondary absorption [8]. The experimental setup is presently based on either a lamp or a laser (as in this paper) as a light source but will eventually involve a battery of fibercoupled LEDs. We are therefore using a lightemission model that is believed to represent the emission from a Lambertian light source such as an LED with a high degree of accuracy [22].

We attempt to quantify and compare the efficiency with which fluorescence EEM spectra of absorbing samples may be collected at different detector configurations. In all configurations, we assume that the excitation light is directed into the sample using a multimode fiber with a realistic emission profile. The fluorescence collection efficiency is modeled using four configurations: (1) detection through the excitation fiber, i.e., collinear but counterpropagating to the excitation beam, (2) detection perpendicular to the excitation beam axis in a finite plane at fixed distance, (3) detection collinear and forward-propagating in a plane at fixed distance from the fiber end face, and (4) detection through six fibers that surround the excitation fiber and have the same numerical aperture and diameter (Fig. 1). It is the aim of this paper to identify the design parameters that need to be adjusted to obtain the largest dynamic concentration range for fluorescence EEM spectroscopy.

\section{Theoretical Model}

We employ a numerical model that is based on geometric (ray) optics, i.e., the wave nature of light is parameterized through Snell's law but not explicitly included. This is justifiable, since in typical 


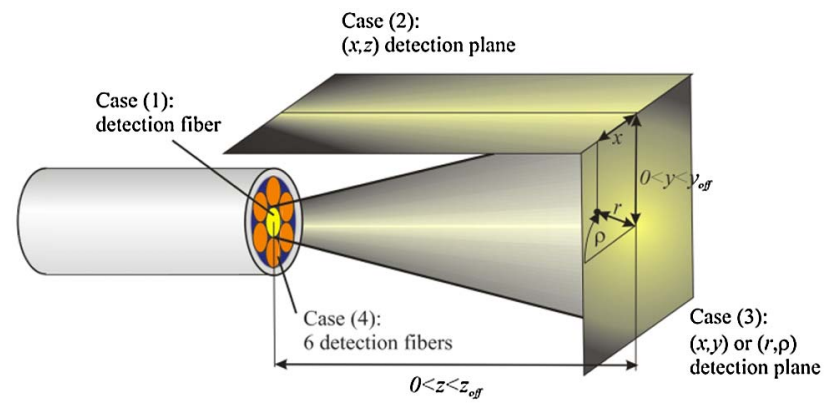

Fig. 1. (Color online) Sketch of the four different detection configurations (see text for details).

fluorescence EEM spectroscopy the dimensions of fibers and detection systems are at least 50 times larger than the wavelength of the light. The excitation of the fluorescence sample is described using the model introduced by $\mathrm{He}$ and Cuomo [22] for multimode-fiber emission. These authors described the emission cone from a multimode fiber-optic cable given its dimensions, numerical aperture (NA), and refractive index of the sample, $n$. Their solutions were verified using their own experiments and are also consistent with the experiments presented here. Instead of providing the lengthy set of equations derived by He and Cuomo, we show the calculated emission of a fiber with a $50 \mu \mathrm{m}$ core into water [Fig. 2(a)]. Figure 2(a) agrees very well with the figures shown in their work, as expected.

Note that $\mathrm{He}$ and Cuomo used a two-dimensional (2D) model to determine the function $I_{\mathrm{exc}}(r, z)$, where $z$ is the distance from the fiber front surface and $r$ is the radial coordinate [22]. The image generated as Fig. 2(a) cannot be compared directly with those obtained using a microscope, however, since imaging of the fluorescence cone is the equivalent of a $2 \mathrm{D}$ projection of the three-dimensional (3D) excitation cone. It is then necessary to, first, generate an Abel transform of the $2 \mathrm{D}$ image, i.e., to generate a cylindrically symmetric three-dimensional (3D) distribution of excitation intensities by rotation of the $2 \mathrm{D}$ distribution of Fig. 2(a) around the center axis, $I_{\text {exc }}(r, \rho, z)$, followed by a projection on the detection plane [Fig. $2(\mathrm{~b})]$ :

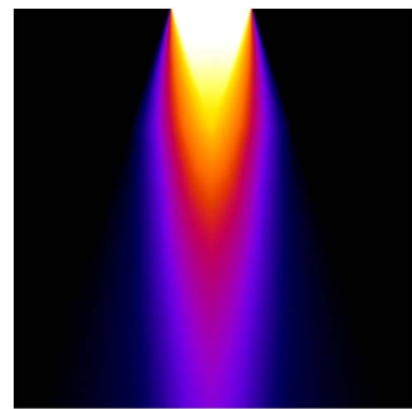

(a)

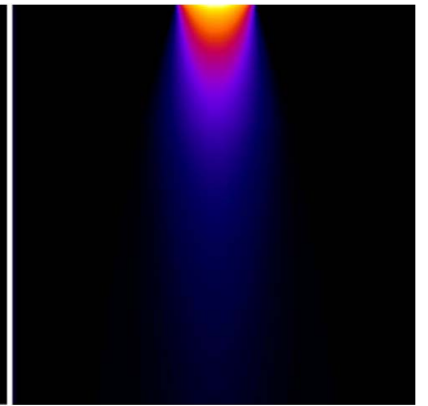

(b)
Fig. 2. (Color online) (a) Two-dimensional representation, $I_{\text {exc }}(r, z)$, of the light intensity emitted from a multimode fiber as calculated using the model by $\mathrm{He}$ and Cuomo [22]. (b) Abel transform of (a), $I_{\text {exc }}(x, z)$, as described in Eq. (1).

$$
\begin{aligned}
I(x, z) & =2 \int_{x}^{\infty} \int_{0}^{2 \pi} I(r, \rho, z) \frac{r}{\sqrt{r^{2}-x^{2}}} \mathrm{~d} \rho \mathrm{d} r \\
& =4 \pi \int_{x}^{\infty} I(r, z) \frac{r}{\sqrt{r^{2}-x^{2}}} \mathrm{~d} r .
\end{aligned}
$$

For most of the cases described below, the cylindrical symmetry is retained and we can simplify our calculations by performing all calculations on the $2 \mathrm{D}$ cross section of the intensity distributions and performing the Abel transform as a final step. Figure 2 shows the excitation cone in the $2 \mathrm{D}$ representation and after Abel transformation of the 3D cylindrically symmetric distribution. It was assumed that the fiber core radius was $r_{0}=50.2 \mu \mathrm{m}$ and the numerical aperture of the fiber was NA $=0.22$, and the refractive index of water is $n$ (water) $=1.33$.

In our model, the excitation cone is further modified by the absorption of the sample, given here as the inverse $1 / e$ absorption length $\alpha_{\text {exc }}\left(\mathrm{cm}^{-1}\right)$. The intensity attenuated by absorption of the excitation beam (primary absorption) is approximated as

$$
\begin{aligned}
I_{\text {prim }}(r, z)= & I_{\mathrm{exc}}(r, z) \\
& \times \begin{cases}\exp \left(-\alpha_{\mathrm{exc}} z\right), & \text { if } r<r_{0}, \\
\exp \left(-\alpha_{\mathrm{exc}} \sqrt{z^{2}+\left(r-r_{0}\right)^{2}},\right. & \text { if } r>r_{0} .\end{cases}
\end{aligned}
$$

The fluorescence emission is assumed to be isotropic, and consequently we can simply determine the probability of detecting the fluorescence from any point in the emission cone by calculating the solid angle formed by the detector plane.

Since the fluorescence collection efficiency depends on the detector configuration, this collection efficiency, $\mathrm{CE}(r, z)$, is the distinguishing term between the four cases that were introduced above. In addition, we need to consider the absorption of the fluorescence by the sample $\alpha_{\mathrm{em}}\left(\mathrm{cm}^{-1}\right)$ ("secondary absorption" or "reabsorption") and the respective absorption length.

For case 1, in which the fluorescence is collected by the same fiber responsible for guiding the excitation light, we use

$$
\begin{aligned}
I_{\text {detect }}(r, z)= & I_{\text {prim }}(r, z) \times \mathrm{CE}(r, z) \\
& \times \begin{cases}\exp \left(-\alpha_{\mathrm{em}} z\right), & \text { if } r<r_{0}, \\
\exp \left(-\alpha_{\mathrm{em}} \sqrt{z^{2}+\left(r-r_{0}\right)^{2}}\right), & \text { if } r>r_{0} .\end{cases}
\end{aligned}
$$

Here the collection efficiency is given by

$$
\mathrm{CE}(r, z)= \begin{cases}\arctan \left(\frac{r+r_{0}}{z}\right)+\arctan \left(\frac{r-r_{0}}{z}\right), & \text { if } r<r_{0}, \\ \arctan \left(\frac{r+r_{0}}{z}\right)-\arctan \left(\frac{r-r_{0}}{z}\right), & \text { if } r>r_{0} .\end{cases}
$$

The total detected intensity is obtained by numerical integration over all $r$ and $z$. Equation (4) assumes that the solid angle of the fluorescence that is captured by the fiber is smaller than the angle of the acceptance cone of the fiber waveguide or, equivalently, that the detection fiber is "underfilled" with 
fluorescence. For our values of NA and $n$, this assumption is valid only at a distance larger than about 3 fiber core diameters. The assumption leads to an overestimate of the collection efficiency at very short distances from the fiber tip. To correct for this effect, the angles calculated by the $\arctan (-)$ expressions in Eq. (4) are limited to a maximum of the critical angle $\Theta_{C}=\arcsin (\mathrm{NA} / n)$ defined by the numerical aperture and the refractive index of the sample.

In case 2 , the detection occurs in a plane that is parallel to the beam axis but offset by a distance $y_{\text {off }}$. Since the detection plane destroys the cylindrical symmetry, we work from the 3D distribution of Eq. (2) and obtain for the projection on the $(x, z)$ detector plane

$$
\begin{aligned}
I_{\text {detect }}(x, z)= & 2 \int_{x}^{\infty} \int_{0}^{2 \pi} I_{\text {prim }}(r, \rho, z) \\
& \times \exp \left[-\alpha_{\mathrm{em}}\left(y_{\text {off }}-r \sin \rho\right)\right] \frac{r}{\sqrt{r^{2}-x^{2}}} \mathrm{~d} \rho \mathrm{d} r .
\end{aligned}
$$

The total detected intensity is obtained by numerical integration of $I(x, z)$ over all $x$ and $z$.

In case 3, we detect fluorescence collinearly and in the forward-propagating direction on a round detector plane at fixed distance, $z_{\text {off }}$, from the fiber end face:

$$
I_{\text {detect }}(r, z)=I_{\text {prim }}(r, z) \mathrm{CE}_{\text {prim }}(r, z) \exp \left[-\alpha_{\mathrm{em}}\left(z_{\text {off }}-z\right)\right] .
$$

The collection efficiency is

$$
\mathrm{CE}(r, z)=\arctan \left(\frac{r_{\text {detect }}+r}{z_{\text {off }}-z}\right)-\arctan \left(\frac{r_{\text {detect }}-r_{0}}{z_{\text {off }}-z}\right) .
$$

As in case 1 , the total detected intensity is obtained by integration over all $r$ and $z$. Here it is assumed that the radius of the detector plane, $r_{\text {detect }}$, is considerably larger than the excitation cone radius at the offset distance.

Finally, case 4 describes the detection through six fibers that surround the excitation fiber and have the same numerical aperture and diameter as the excitation fiber. The fluorescence intensity that falls into six fibers placed in a ring with outer radius $2 r_{\mathrm{cl}}$ and inner radius $r_{\mathrm{cl}}$ can be calculated as
As in case 1 , the angles calculated from the $\arctan (-)$ functions are restricted to be smaller than the critical incidence angle, $\Theta_{C}$. The term $\Gamma(r, z)$ takes into consideration that the collection area offered by six fibers placed inside a ring increases from zero at the inner rim of the ring to a maximum near the center of the rim. The function $\Gamma(r, z)$ is defined between the inner and outer rim of the ring and can be derived as

$$
\Gamma(r, z)=\frac{6}{\pi} \arctan \left\{\frac{\sqrt{16 r_{\mathrm{cl}}^{2} r^{2}-\left[4 r_{\mathrm{cl}}^{2}-r_{0}^{2}(z)+r^{2}\right]^{2}}}{4 r_{\mathrm{cl}}^{2}-r_{0}^{2}(z)+r^{2}}\right\} .
$$

The width of the ring increases with distance $z$ from the fiber ends according to the numerical aperture and the refractive index of the sample. This is expressed by

$$
r_{0}(z)=r_{0}(0)\left(1+\frac{z}{\sqrt{n^{2} / \mathrm{NA}^{2}-1}}\right) .
$$

More information on the derivation of Eq. (10) is given in the Appendix.

\section{Experiment}

Fluorescence emission from a multimode fiber (OFS Specialty Photonics Division, USA; core diameter $50 \mu \mathrm{m}$, cladding diameter $125 \mu \mathrm{m}, \mathrm{NA}=0.2$ ) was imaged using a microscope (Axio Imager.M2m with EC Epiplan-Neofluar 10x objective, Carl Zeiss MicroImaging, Germany). The fiber was submersed in a petri dish containing solutions of the dye Eosin Y (BASF, Germany; used as supplied) at five different concentrations between 0.857 and $0.0535 \mathrm{mM}$ in basic ethanol (96\%, Carl Roth; used without further purification). The images were recorded in 8 bit linear gray scale and processed using Image-J software (W. Rasband, National Institute of Health, USA). The imaging modus therefore corresponds to "case 2 ," but a quantitative analysis was not attempted, since the absorption path through the sample, $y_{\text {off }}$ in Eq. ()), can only be estimated as $2-4 \mathrm{~mm}$. The

$$
I_{\text {detect }}(r, z)= \begin{cases}I_{\text {prim }}(r, z) \times C E_{A}(r, z) \times \exp \left(-\alpha_{\mathrm{em}} \sqrt{z^{2}+\left(r-2 r_{\mathrm{cl}}\right)^{2}}\right), & \text { if } r>2 r_{\mathrm{cl}}+r_{0}, \\ I_{\text {prim }}(r, z) \times C E_{B}(r, z) \times \exp \left(-\alpha_{\mathrm{em}} \sqrt{z^{2}+\left(r_{\mathrm{cl}}-r\right)^{2}}\right), & \text { if } r<2 r_{\mathrm{cl}}-r_{0}, \\ I_{\text {prim }}(r, z) \times C E_{C}(r, z) \times \exp \left(-\alpha_{\mathrm{em}} z\right), & \text { if } 2 r_{\mathrm{cl}}-r_{0}<r<2 r_{\mathrm{cl}}+r_{0} .\end{cases}
$$

The collection efficiency of six fibers with core radius $r_{0}$ and cladding radius $r_{\mathrm{cl}}$ can be calculated as

$$
\begin{aligned}
& \mathrm{CE}_{A}(r, z)=\Gamma(r, z) \times\left(\arctan \left(\frac{r-2 r_{\mathrm{cl}}-r_{0}}{z}\right)-\arctan \left(\frac{r-2 r_{\mathrm{cl}}+r_{0}}{z}\right)\right), \quad \text { if } r>2 r_{\mathrm{cl}}+r_{0} ; \\
& \mathrm{CE}_{B}(r, z)=\Gamma(r, z) \times\left(\arctan \left(\frac{2 r_{\mathrm{cl}}-r_{0}-r}{z}\right)-\arctan \left(\frac{2 r_{\mathrm{cl}}+r_{0}-r}{z}\right)\right), \quad \text { if } r<2 r_{\mathrm{cl}}-r_{0} ; \\
& \mathrm{CE}_{C}(r, z)=\Gamma(r, z) \times\left(\arctan \left(\frac{2 r_{\mathrm{cl}}+r_{0}-r}{z}\right)+\arctan \left(\frac{r-2 r_{\mathrm{cl}}+r_{0}}{z}\right)\right), \quad \text { if } 2 r_{\mathrm{cl}}-r_{0}<r<2 r_{\mathrm{cl}}+r_{0} .
\end{aligned}
$$


images are therefore used primarily to check the validity of the model expressed by Eqs. (1)-( $\underline{4})$.

\section{Results of the Theoretical Models}

To determine the dynamic intensity range for the EEM experiments, we made a number of assumptions. First, we assume that at all wavelengths both the fluorescence quantum efficiency and the detector response are unity, and that optical loss at the fibersample interface is negligible. These assumptions only introduce constant factors into the equations.

We have selected the absorption coefficients of the fluorescent dye Eosin $\mathrm{Y}$ in our models, since this allows us to compare our model with experiments. At the excitation wavelength of $532 \mathrm{~nm}$, the molar extinction coefficient of the sample based on the decadic logarithm, $\varepsilon$, is $86,400 \mathrm{lmol}^{-1} \mathrm{~cm}^{-1}$ [23,24], and at the fluorescence maximum of $543.5 \mathrm{~nm}, \varepsilon=$ $24,500 \mathrm{lmol}^{-1} \mathrm{~cm}^{-1}$.

For this dye and many others in which the fluorescence maximum is not greatly shifted from the absorption maximum, the fluorescence emission following excitation to strong absorption bands in the UV is also more strongly attenuated compared with fluorescence excited and emitted at longer wavelengths. This assumption is therefore consistent with the frequent observation that strong primary absorption is accompanied by strong secondary absorption and that weak primary absorption is accompanied by weak secondary absorption. The simulated dye concentration is varied in 18 steps between $430 \mathrm{nM}$ and $0.11 \mathrm{M}$, and the refractive index is set to that of ethanol ( $n=1.362$ ), whereas the numerical aperture of the $50 / 125 \mu \mathrm{m}$ multimode fiber is set to $\mathrm{NA}=0.2$.

At all concentrations we calculate the $2 \mathrm{D}$ image, $I_{\text {prim }}(r, z)$, of the excitation light after primary absorption and the probability that a particular volume element contributes to detected fluorescence, $I_{\text {detect }}(r, z)$. These images are shown in Fig. $\underline{3}$ for Eosin Y concentration of 3 and $440 \mu \mathrm{M}$ and for all four cases. Figure 3(a) shows the effect of primary absorption for both concentrations. It is apparent that at a concentration of $440 \mu \mathrm{M}$, a strongly absorbing dye absorbs (and fluoresces) largely within $100 \mu \mathrm{m}$ of the fiber surface, which is consistent with the 1/e absorption length of this dye at $532 \mathrm{~nm}$ of $d=260 \mu \mathrm{m}$. All other panels show the probability that point $(r, z)$ contributes to the detected fluorescence intensity according to Eq. (3). The "brightness" is a product of the intensity of the excitation beam, the primary and secondary absorption, and the solid angle for the fluorescence detection. The images for cases 1-3 show that the largest contribution to the detected fluorescence arises from a volume within about 100-300 $\mu \mathrm{m}$ of the fiber end. This is true for all samples regardless of their absorption coefficients. Case 4 is unusual, since in a fiber bundle (or in a bifurcated fiber) the volume in front of the excitation fiber cannot contribute to the detected fluorescence (Fig. 4). The overall intensity then depends strongly on the mode overlap of the collection fiber(s) with the
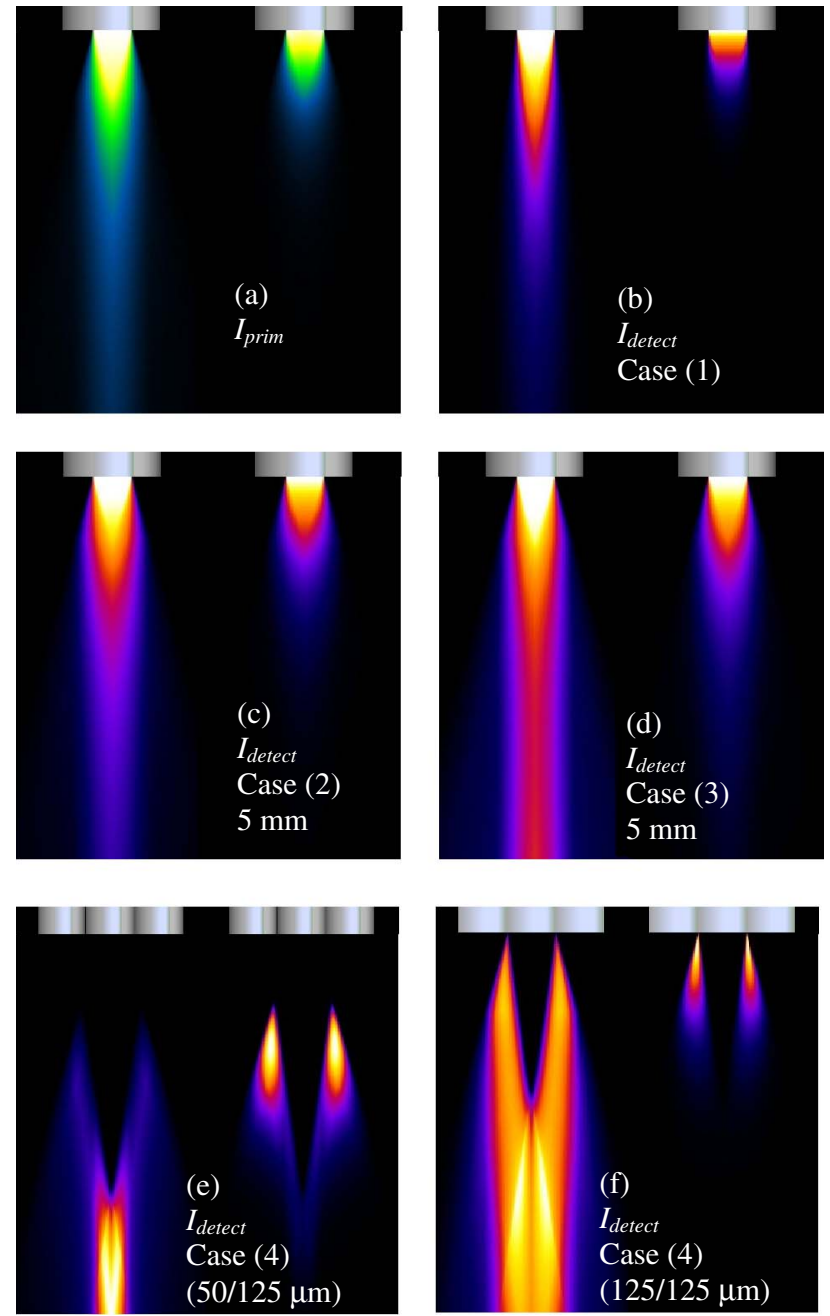

Fig. 3. (Color online) False-color 2D cross section of the spatial distribution of light following absorption and emission from a multimode fiber with a $50 \mu \mathrm{m}$ core into Eosin Y dye solution in basic ethanol. The left images assume an Eosin Y concentration of $3 \mu \mathrm{M}$, and the right images assume $440 \mu \mathrm{M}$. Intensities have been rescaled to cover the entire dynamic range of the image. (a) Primary absorption of excitation light calculated using the model by $\mathrm{He}$ and Cuomo [22]. (b) Fluorescence collection probability assuming that the excitation fiber also collects fluorescence (case 1). (c) Fluorescence collection probability assuming that light is collected by a detector placed $5 \mathrm{~mm}$ above the image plane (case 2). (d) Fluorescence collection probability assuming that a detector looking into the fiber is placed $5 \mathrm{~mm}$ "downstream" of the light emission (case 3). (e)-(f) Fluorescence collection probability of two fibers aligned parallel to the center emission fiber, where the emission and collection fibers have (e) a $50 \mu \mathrm{m}$ core and $125 \mu \mathrm{m}$ cladding diameter or (f) a $125 \mu \mathrm{m}$ core and no cladding (F) (case 4).

excitation fiber, i.e., on their distance, the thickness of the cladding, the numerical aperture, and the refractive index of the sample. As is illustrated in Fig. 4 , changing the fiber from a $50 / 125 \mu \mathrm{m}$ multimode fiber to a claddingless $125 \mu \mathrm{m}$ fiber reduces the "dead volume" in front of the excitation fiber. This is important because almost the entire fluorescence excitation cone (Fig. 2) falls into the "dead volume," and this effect is responsible for the sharp drop-off in 


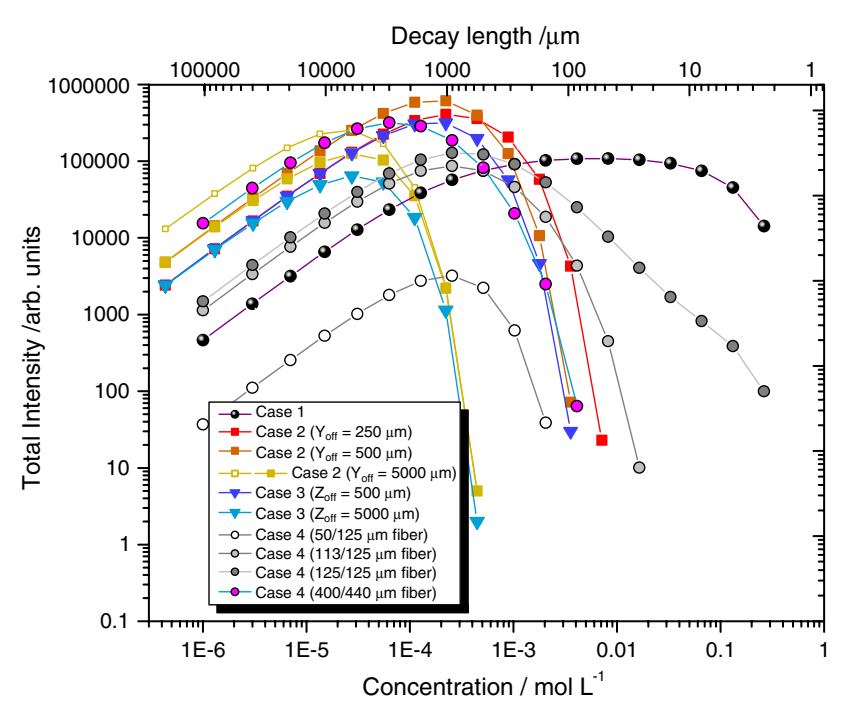

Fig. 4. (Color online) Calculated total intensity for the four different cases. The simulation assumes $\mathrm{NA}=0.20$ and $n_{\text {solution }}=$ 1.362 and absorption coefficients of Eosin $\mathrm{Y}$ dye at the excitation wavelength of $532 \mathrm{~nm}\left(\varepsilon=86,400 \mathrm{lmol}^{-1} \mathrm{~cm}^{-1}\right)$ and at the center of the emission spectrum at $543.5 \mathrm{~nm}\left(\varepsilon=24,500 \mathrm{lmol}^{-1} \mathrm{~cm}^{-1}\right)$. The intensity of a cube with $500 \mu \mathrm{m}$ sides is integrated except in case $2\left(y_{\text {off }}=5000 \mu \mathrm{m}\right.$; yellow squares $)$ when the length of the region was $2.5 \mathrm{~mm}$, in case 4 (113 and $125 \mu \mathrm{m}$ core) when the sides of the cube were $1 \mathrm{~mm}$ long, and in case 4 (400 $\mu \mathrm{m}$ core) when the sides were $4 \mathrm{~mm}$ long.

detected fluorescence of strongly absorbing samples by fiber-bundle probes.

Figure 4 shows even more clearly that sampling in front of the excitation fiber is essential for measurements when primary and secondary absorption is high. The figure displays the total integrated intensity calculated from the Abel-transformed images [Fig. 2(b)] and is shown as a function of the Eosin $\mathrm{Y}$ concentration. As expected, the collection efficiency of all detector configurations drops sharply when the absorption decay length is less than about $100 \mu \mathrm{m}$. The one exception is detection through the same fiber that is used for excitation as in case 1 . A counterpropagating detection with claddingless fibers is also favorable at short absorption decay lengths (case 4 with $125 / 125 \mu \mathrm{m}$ fibers). A fiber bundle made with fibers of intermediate cladding diameter $(113 / 125 \mu \mathrm{m})$ shows a lower range of detection, whereas a bundle made from $50 / 125 \mu \mathrm{m}$ fibers has the lowest overall fluorescence collection. This is partly due to the reduced throughput through the smaller fiber core, but also due to the much larger dead volume.

The drop of collected fluorescence at low concentrations is partly due to an artifact of the modeling, since the integration volume is typically restricted to a cube of $500 \mu \mathrm{m}$ length to reduce the computational time-except for case 4 , where it is $1 \mathrm{~mm}$ for fibers of 113 and $125 \mu \mathrm{m}$ core diameter and $4 \mathrm{~mm}$ for the $400 \mu \mathrm{m}$ core fiber. Should the absorption be very weak and the fluorescing sample volume extend over several millimeters, the collected fluorescence intensity is therefore underrepresented. To estimate this

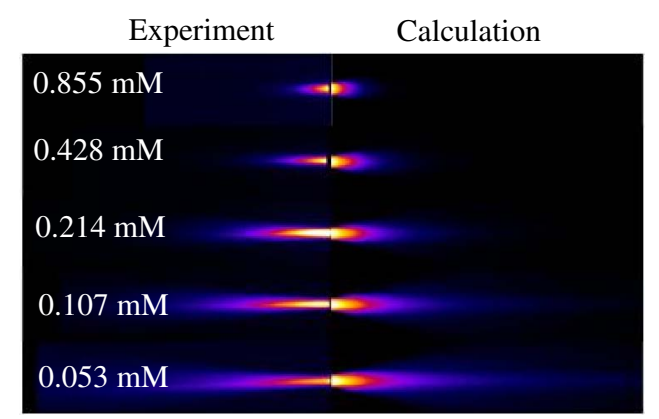

Fig. 5. (Color online) Eosin Y fluorescence images. Left: falsecolor images detected through a microscope. The concentrations correspond to a dilution series of a $0.857 \mathrm{mM}$ solution to $1: 2$, 1:4, 1:8 and 1:16 (top to bottom). Right: simulated fluorescence profiles using case 2 and $y_{\text {off }}=3 \mathrm{~mm}$. The absorption coefficients were the same as in Fig. 4. Each of the 10 images is $500 \mu \mathrm{m}$ high.

effect, we performed one calculation of a $0.5 \mathrm{~mm} \times$ $0.5 \mathrm{~mm} \times 2.5 \mathrm{~mm}$ volume and include the result in Fig. 5. Note that this inaccuracy does not affect the main result of the calculation: all configurations are similarly suited to detect fluorescence when the sample is not strongly absorbing, but at high optical density, only two configurations perform well, i.e., the fiber probes in which either the excitation fiber itself or a nearby claddingless fiber collects the fluorescence.

\section{Consistency of the Model with Experimental Observations}

The intensity distribution of the excitation light and consequently also of the fluorescence is described using the model by $\mathrm{He}$ and Cuomo [22]. Since this model deviates from those used by previous researchers and in particular from what is found in textbooks, we attempted to experimentally verify its validity.

The false-color photographs recorded through a microscope show an intensity distribution that agrees qualitatively with that expected using our case 2 scenario, i.e., projection onto a detection plane after performing the Abel transform (Fig. 5). The decay lengths calculated from the images also agree within 10\%-20\% with what is expected using the known absorption cross section and the calculated images using case 2 . We can therefore conclude that for the present 50-125 $\mu \mathrm{m}$ multimode fiber, the model by $\mathrm{He}$ and Cuomo represents a sufficiently accurate description of the excitation cone. We note in particular that the radial distribution very close to the fiber is described by a flat-topped intensity distribution, whereas at a distance that is long compared with the diameter of the fiber core, the beam evolves into a profile that resembles a Gaussian function. One of the key assumptions of He and Cuomo's model is that the fiber is illuminated by a Lambertian source, i.e., the uniangular beams entering the fiber have identical power. In contrast, we coupled light from a laser (beam diameter $\sim 2 \mathrm{~mm}$ ) into the fiber using an $f=20 \mathrm{~mm}$ lens, i.e., the acceptance cone was not filled entirely. To verify that the 
deviations between the observed and calculated fluorescence images are due to incomplete mode scrambling in our $1 \mathrm{~m}$ fiber cable, we recorded images similar to those shown in Fig. 5 but using a diffuser in front of the entrance face of the fiber. These images, as well as those recorded without a lens at normal incidence and at $17^{\circ}$ and $56^{\circ}$ angles, show fluorescence cones of slightly different width. This supports our assumption that the modes were not effectively scrambled in the fiber and that the difference between model and experiment are due to the non-Lambertian laser light source. For an accurate determination of the specific collection efficiency, one therefore may also have to consider the extent of mode scrambling and the emission characteristics of the excitation light source. We estimate that mode scrambling may require several tens or hundreds of meters of optical fiber [25]. Other models for the light emission from a multimode fiber may then be appropriate but are not expected to invalidate the conclusions of this paper.

\section{Discussion}

Our comparison of the four different fluorescence detection schemes in combination with the experimental observations allows us to make the following statements: (i) Collecting fluorescence through the same waveguide that is used for fluorescence excitation permits the largest dynamic range of concentration measurements and in a fluorescence EEM image is expected to yield the data array with the largest information content. (ii) Because of the dead volume, the use of a fiber bundle to excite and collect fluorescence may limit the ability to record fluorescence from strongly absorbing samples. (iii) The dead volume can be reduced by using either claddingless fibers for fluorescence excitation and detection or, as described by Booksh and coworkers, a coaxial capillary collection waveguide with a claddingless excitation fiber [14]. (iv) With regard to dynamic range, the common method of detecting fluorescence normal to the excitation axis $[18,19,26]$ is inferior to any of the fiber-coupled methods when the thickness of the sample cuvette is larger than the approximate decay length of the secondary absorption. (v) Collecting fluorescence in transmission-as is still common in microscopy ("trans-fluorescence detection") $[27,28]$ is similarly (in-) efficient compared with collection at right angles. Of course, this method requires that the detection system is capable of separating the fluorescence from the excitation light.

The effect of the dead volume in front of the excitation fiber was described in some detail before and gave rise to a number of improvements, including the use of double-clad fibers [14,15], beveling the fiber ends [2,11-13], using high-N fiber [20], and the use of lenses $[11,12]$. Indeed, the Monte Carlo simulation by Ozanyan et al. [20] generated collection efficiency data for a single-fiber configuration and a bifurcated fiber arrangement that are qualitatively and quantitatively very similar to the curves shown in Fig. 4 for case 1 and case 4 . Despite the different models for the light emission and the differences in calculating the collection efficiencies, both curves show a steep decrease of the fluorescence collection efficiency when the absorption length is of the order of the fiber cladding diameter.

While the use of a single fiber therefore appears to be superior to any of the other fluorescence collection schemes, the difficulty of separating the-sometimes weak-fluorescence from the intense excitation light has prompted many instrument manufacturers and research groups to instead use a fiber bundle. A compromise may therefore be the use of a transparent silica or quartz spacer in front of the bundle. In the spacer, the acceptance cones of the excitation and emission fibers overlap such that the fluorescence, which is generated in the sample and irradiates the front surface of the spacer, falls into the acceptance cones of the collection fibers. Ideally such a spacer would have a radius of $2 r_{\mathrm{cl}}+r_{0}$ and a length of at least twice that of the dead volume. We note that the importance of distance from an emitting or reflecting sample surface when using a fiber-bundle probe has already been highlighted, for example, by Papaioannou et al., in the discussion of the optimization of fiber probes for tissue analysis [16], whose assessment is strongly supported by the present study.

Finally, the use of fiber lenses to collimate the excitation light may be considered as a means to enhance the collection efficiency. While a detailed investigation is beyond the scope of this article, one may expect that the effect of collimation is only minimal in the scenarios described by case 1 , case 2 , and case 3 , since in these cases the entire illumination volume is probed by the collection fiber, as in case 1 , or area detectors, as in cases 2 and 3 . It is therefore largely irrelevant if the shape of the irradiated volume changes. In case 4 , collimation of light will have a detrimental effect, since it will further increase the dead volume. In this case, one may even consider the use of a diverging fiber lens to enable the fluorescence detection of strongly (re-) absorbing samples.

\section{Appendix A: Calculation of the Collection Efficiency of a Fiber Bundle}

It is assumed that a single excitation fiber with core radius $r_{0}$ and cladding radius $r_{\mathrm{cl}}$ is surrounded by six fibers with identical core and cladding radii. Close to the fiber ends, the fluorescence will be excited in front of the center fiber core but will not be collected by the six surrounding fibers. At a larger distance, the fluorescence has diverged somewhat and the collection efficiency, CE, asymptotically approaches that of case 1 as expressed by Eq. (4).

If a capillary [14] or double-clad fiber [15] is used to collect fluorescence, we obtain $\Gamma=1$, i.e., all fluorescence falling into the ring defined by the two red circles in Fig. S1 is detected. The six fibers cover a smaller detector area compared with the "detection 


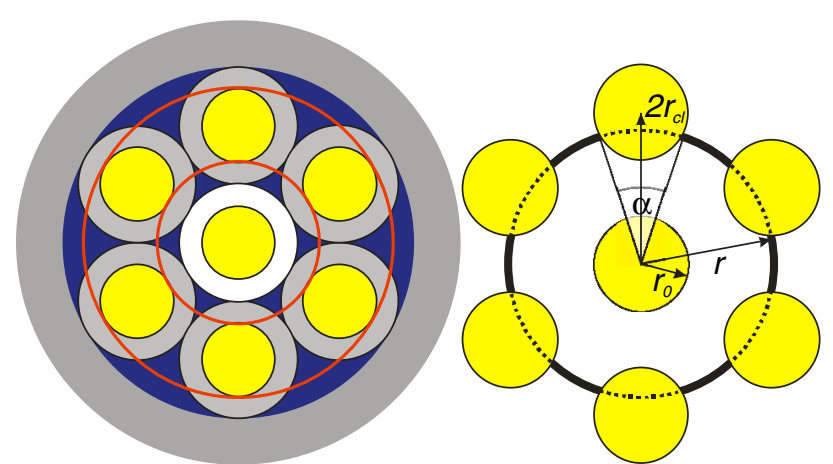

Fig. 6. (Color online) Geometry of the 50/125 $\mu$ m fiber bundle.

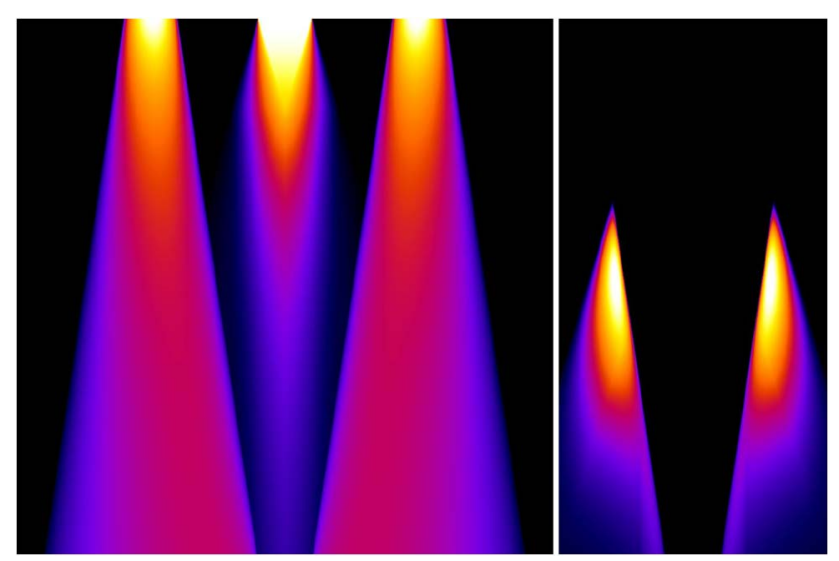

Fig. 7. (Color online) Left, collection efficiency of two fibers together with the fiber excitation cone (center); right, resulting fluorescence collection assuming a $440 \mu \mathrm{M}$ concentration of Eosin $\mathrm{Y}$ [akin to Fig. 4(a)].

ring," though, and the deviation from the above collection efficiency, $\Gamma(r, z)$ has to be included.

We assume that a point emitting light at a distance $r$ from the beam axis has a probability of contributing to the signal that corresponds to the fraction of the circle segments (Fig. $\underline{6}$, dotted lines) relative to $2 \pi$ :

$$
\Gamma=6 \alpha(r, z) / 2 \pi \text {. }
$$

The angle associated with the circle segments is calculated from the equation of overlapping circles:

$$
\alpha(r, z)=2 \arctan \left\{\frac{\sqrt{16 r_{\mathrm{cl}}^{2} r^{2}-\left[4 r_{\mathrm{cl}}^{2}-r_{0}^{2}(z)+r^{2}\right]^{2}}}{4 r_{\mathrm{cl}}^{2}-r_{0}^{2}(z)+r^{2}}\right\} .
$$

The probability of falling into the acceptance cone of any of the six fibers also increases with distance, and this is incorporated into Eq. (A2) by modifying the acceptance radius of the collection fiber according to the refractive index of the sample, $n$, and the numerical aperture of the fiber, NA:

$$
r_{0}(z)=r_{0}(0)\left(1+\frac{z}{\sqrt{n^{2} / \mathrm{NA}^{2}-1}}\right) .
$$

By combining Eq. (9) with Eqs. (A1)-(A3), we obtain collection efficiencies as shown in Fig. 7 (left). Together with the calculated emission cone of the central fiber (also shown in Fig. $\underline{7}$, left), it is then possible to determine the contribution of each volume element to the detected fluorescence (Fig. $\underline{7}$, right).

H.-P. Loock, J. Saunders, and H. Omrani thank GasTOPS Ltd. (Ottawa, ON, Canada) and the Natural Sciences and Engineering Research Council of Canada for financial support. D. Munzke, J. Saunders, O. Reich, and H.-P. Loock acknowledge the financial support of the German Federal Ministry of Education and Research (grant 03Z2AN12). J. Saunders thanks the Canadian Institute for Photonic Innovations for partial financial support of his visit to innoFSPEC.

\section{References}

1. A. G. Mignani, L. Ciaccheri, C. Cucci, A. A. Mencaglia, A Cimato, C. Attilio, H. Ottevaere, H. Thienpont, R. Paolesse, M. Mastroianni, D. Monti, M. Gerevini, G. Buonocore, M. A. Del Nobile, A. Mentana, M. F. Grimaldi, C. Dall'Asta, A. Faccini, G. Galaverna, and A. Dossena, "EAT-by-LIGHT: fiberoptic and micro-optic devices for food quality and safety assessment," IEEE Sens. J. 8, 1342-1354 (2008).

2. E. V. Trujillo, D. R. Sandison, U. Utzinger, N. Ramanujam, M. F. Mitchell, and R. Richards-Kortum, "Method to determine tissue fluorescence efficiency in vivo and predict signalto-noise ratio for spectrometers," Appl. Spectrosc. 52, 943-951 (1998).

3. N. Ramanujam, M. Follen Mitchell, A. Mahadevan-Jansen, S. L. Thomsen, G. Staerkel, A. Malpica, T. Wright, N. Atkinson, and R. Richards-Kortum, "Cervical precancer detection using a multivariate statistical algorithm based on laser-induced fluorescence spectra at multiple excitation wavelengths," Photochem. Photobiol. 64, 720-735 (1996).

4. T. J. Pfefer, K. T. Schomacker, and N. S. Nishioka, "Effect of fiber optic probe design on fluorescent light propagation in tissue," Proc. SPIE 4257, 410-416 (2001).

5. M. L. Nahorniak and K. S. Booksh, "Excitation-emission matrix fluorescence spectroscopy in conjunction with multiway analysis for PAH detection in complex matrices," Analyst 131, 1308-1315 (2006).

6. A. G. Mignani, L. Ciaccheri, N. Díaz-Herrera, A. A. Mencaglia, H. Ottevaere, H. Thienpont, S. Francalanci, A. Paccagnini, and F. S. Pavone, "Optical fiber spectroscopy for measuring quality indicators of lubricant oils," Meas. Sci. Technol. 20, 034011 (2009).

7. K. R. Rogers and E. J. Poziomek, "Fiber optic sensors for environmental monitoring," Chemosphere 33, 1151-1174 (1996).

8. H. Omrani, J. A. Barnes, A. E. Dudelzak, H.-P. Loock, and H. Waechter, "Fluorescence excitation-emission matrix (EEM) spectroscopy and cavity ring-down (CRD) absorption spectroscopy of oil-contaminated jet fuel using fiber-optic probes," Analyst 137, 2782-2790 (2012).

9. J. J. Ma, Y. Chiniforooshan, W. H. Hao, W. J. Bock, and Z. Y. Wang, "Easily fabricated, robust fiber-optic probe for weak fluorescence detection: modeling and initial experimental evaluation," Opt. Express 20, 4805-4811 (2012).

10. P. Plaza, Q. D. Nguyen, M. Jouan, H. Fevrier, and H. Saisse, "Simulation and optimization of adjacent optical fiber sensors," Appl. Opt. 25, 3448-3454 (1986).

11. T. F. Cooney, H. T. Skinner, and S. M. Angel, "Comparative study of some fiber-optic remote Raman probe designs. Part I: model for liquids and transparent solids," Appl. Spectrosc. 50, 836-848 (1996).

12. T. F. Cooney, H. T. Skinner, and S. M. Angel, "Comparative study of some fiber-optic remote Raman probe designs. Part 
II: tests of single-fiber, lensed, and flat- and bevel-tip multifiber probes," Appl. Spectrosc. 50, 849-860 (1996).

13. A. Blanco, E. Chomski, S. Grabtchak, M. Ibisate, S. John, S. W. Leonard, C. Lopez, F. Meseguer, H. Miguez, J. P. Mondia, G. A. Ozin, O. Toader, and H. M. van Driel, "Large-scale synthesis of a silicon photonic crystal with a complete three-dimensional bandgap near 1.5 micrometres," Nature 405, 437-440 (2000).

14. Y. C. Kim, J. A. Jordan, D. Chavez, and K. S. Booksh, "Coaxial fiber-optic chemical-sensing excitation-emission matrix fluorometer," Opt. Lett. 36, 355-357 (2011).

15. L. Wang, H. Y. Choi, Y. M. Jung, B. H. Lee, and K. T. Kim, "Optical probe based on double-clad optical fiber for fluorescence spectroscopy," Opt. Express 15, 17681-17689 (2007).

16. T. Papaioannou, N. W. Preyer, Q. Y. Fang, A. Brightwell, M. Carnohan, G. Cottone, R. Ross, L. R. Jones, and L. Marcu, "Effects of fiber-optic probe design and probe-to-target distance on diffuse reflectance measurements of turbid media: an experimental and computational study at $337 \mathrm{~nm}$," Appl. Opt. 43, 2846-2860 (2004).

17. G. K. Bhowmick, N. Gautam, and L. M. Gantayet, "Design optimization of fiber optic probes for remote fluorescence spectroscopy," Opt. Commun. 282, 2676-2684 (2009).

18. W. D. Kulatilaka, P. S. Hsu, J. R. Gord, and S. Roy, "Point and planar ultraviolet excitation/detection of hydroxyl-radical laser-induced fluorescence through long optical fibers," Opt. Lett. 36, 1818-1820 (2011).

19. P. S. Hsu, W. D. Kulatilaka, N. B. Jiang, J. R. Gord, and S. Roy, "Investigation of optical fibers for gas-phase, ultraviolet laserinduced-fluorescence (UV-LIF) spectroscopy,” Appl. Opt. 51, 4047-4057 (2012).
20. K. B. Ozanyan, T. L. Yeo, F. P. Hindle, N. R. J. Poolton, H. McCann, and K. L. Tan, "Fiber-based UV laser-diode fluorescence sensor for commercial gasolines," IEEE Sens. J. 4, 681-690 (2004).

21. T. J. Pfefer, A. Agrawal, and R. A. Drezek, "Oblique-incidence illumination and collection for depth-selective fluorescence spectroscopy," J. Biomed. Opt. 10, 044016 (2005).

22. G. He and F. W. Cuomo, "A light-intensity function suitable for multimode fiberoptic sensors," J. Lightwave Technol. 9, 545-551 (1991).

23. J. M. Dixon, M. Taniguchi, and J. S. Lindsey, "PhotochemCAD 2: a refined program with accompanying spectral databases for photochemical calculations," Photochem. Photobiol. 81, 212-213 (2005).

24. H. Du, R. C. A. Fuh, J. Z. Li, L. A. Corkan, and J. S. Lindsey, "PhotochemCAD: a computer-aided design and research tool in photochemistry," Photochem. Photobiol. 68, 141-142 (1998).

25. W. F. Rong, Y. X. Wang, T. Y. Chai, Y. K. Yeo, M. T. Zhou, and T. H. Cheng, "Bandwidth enhancement of multimode fiber based on a cost-effective periodic mode-scrambling method," Opt. Eng. 47, 115002 (2008).

26. J. R. Lakowicz, Principles of Fluorescence Spectroscopy, 3rd ed. (Springer, 2006), Chap. 5.

27. A. Larson, V. Iyer, T. Hoogland, and P. Saggau, "Fiber-coupled non-descanned $4 \pi$ detection with a commercial confocal microscope modified for multiphoton imaging," Proc. SPIE 4963 239-251 (2003).

28. P. T. Tran and F. Chang, "Transmitted light fluorescence microscopy revisited," Biol. Bull. 201, 235-236 (2001). 